\title{
ASSESSMENT OF FEMORAL NECK STRENGTH AND BONE MINERAL DENSITY CHANGES FOLLOWING EXERCISE USING 3D-DXA IMAGES
}

Dermot O'Rourke

Medical Device Research Institute, College of Science and Engineering, Flinders University, Australia

1284 South Road, Clovelly Park, SA 5042, Australia

dermot.orourke@flinders.edu.au

Belinda R Beck

Menzies Health Institute Queensland, Griffith University, Gold Coast, Australia

School of Allied Health Sciences, Griffith University, Gold Coast, Australia

The Bone Clinic, Brisbane, Australia

Amy T Harding

Menzies Health Institute Queensland, Griffith University, Gold Coast, Australia

School of Allied Health Sciences, Griffith University, Gold Coast, Australia

Steven L Watson

Menzies Health Institute Queensland, Griffith University, Gold Coast, Australia

School of Allied Health Sciences, Griffith University, Gold Coast, Australia

Peter Pivonka

School of Mechanical, Medical and Process Engineering, Queensland University of Technology, Brisbane, Australia.

Saulo Martelli

School of Mechanical, Medical and Process Engineering, Queensland University of Technology, Brisbane, Australia.

Medical Device Research Institute, College of Science and Engineering, Flinders University, Australia

Word Count (Introduction through Discussion): 3,396 


\section{KEYWORDS (5 words)}

Exercise, strength, BMD, DXA, femur

\section{ABSTRACT (250 words)}

Physical exercise induces spatially heterogeneous bone changes in the proximal femur. Recent advances have enabled 3D dual-energy X-ray Absorptiometry (DXA)-based finite element (FE) models to estimate bone strength. However, its ability to detect exercise-induced BMD and strength changes is unclear. The aim of this study was to quantify the repeatability of vBMD and femoral neck strength obtained from 3D-DXA images and determine the changes due an exercise intervention. The DXA scans included pairs of same-day repeated scans from ten healthy females and pre- and post-exercise intervention scans of 26 males. FE models with element-by-element correspondence were generated by morphing a template mesh to each bone. BMD and femoral strength under single-leg-stance and sideways fall loading configurations were obtained for both groups and compared. In the repeated images, the total hip vBMD difference was $0.5 \pm 2.5 \%$. Element-by-element BMD differences reached $30 \pm$ $50 \%$. The strength difference in single-leg stance was $2.8 \pm 13 \%$ and in sideways fall was $4.5 \%$ $\pm 19 \%$. In the exercise group, strength changes were $6 \pm 19 \%$ under single-leg stance and $1 \pm$ $18 \%$ under sideways fall. vBMD parameters were weakly correlated to strength $\left(\mathrm{R}^{2}<0.31\right)$. The exercise group had a mean bone accrual exceeding repeatability values in the femoral head and cortical regions. The case with the highest vBMD change $(6.4 \%)$ caused $18 \%$ and $-7 \%$ strength changes under single-leg stance and sideways fall. 3D-DXA technology can assess the effect of exercise interventions in large cohorts but its validity in individual cases should be interpreted with caution. 


\section{INTRODUCTION}

Osteoporosis is an age-related disease characterised by decreased bone mineral density (BMD) and increased risk of fracture after minimal trauma. Osteoporotic hip fractures are associated with a high burden of morbidity, healthcare costs, and mortality (Abrahamsen et al., 2009; Johnell and Kanis, 2006). Physical exercise is recommended for mitigating the effects of age-related bone loss (Beck et al., 2017) as it causes higher than normal strain in bone which elicits an adaptive skeletal response (Rubin and Lanyon, 1985). Typically, the bone response to exercise is assessed using areal Bone Mineral Density (aBMD) calculated over large bone portions. However, few data exist on spatially heterogeneous bone changes in response to exercise (Lang et al., 2014) and even fewer on the relationship of such changes to alterations in femoral neck strength.

According to the World Health Organization (WHO), the assessment of the risk of hip fracture is based on population-based aBMD values measured using dual-energy X-ray Absorptiometry (DXA) imaging. The process involves calculating the bone area and mass over the total hip, neck, trochanter, and sub-trochanter region from planar DXA images. However, aBMD obtained from DXA has shown to discriminate fracture cases from non-fracture with area under the curve (AUC) values of approximately 0.75 (Qasim et al., 2016; Yang et al., 2009) and been only weakly associated with bone strength $\left(\mathrm{R}^{2}=0.3-0.69\right)$ (Cheng et al., 1997; Taddei et al., 2014), motivating research into advanced diagnostic methods. Recent technologies have used finite-element modelling based on 3D femur geometry and the volumetric BMD (vBMD) distribution derived from computed-tomography $(\mathrm{CT})$ images to estimate bone strength. These CT-based models predict femoral neck strength better than standard empirical relationships using aBMD, $\left(\mathrm{R}^{2}=0.8, \mathrm{SEE}=1314 \mathrm{~N}\right.$ vs $\mathrm{R}^{2}=0.66, \mathrm{SEE}=$ 1687N) (Dall'Ara et al., 2016), and are better able to identify clinical fracture and non-fracture cases (Falcinelli et al., 2014). However, the dose of ionizing radiation in clinical CT scanners 
limits the applicability of CT-based methods in large clinical trials and in routine clinical practice.

Recent advances of image processing technologies have enabled reconstructing the 3D geometry and BMD distribution from planar DXA images, referred to as the 3D-DXA images hereinafter, and studying volumetric bone density and strength changes following clinical interventions. One viable procedure embedded in the 3D-SHAPER (Galgo Medical, Barcelona, Spain) software, involves extracting from a statistical shape and appearance model (SSAM) of hip geometry and bone mineral density the instance that best-matches the DXA image from the patient, providing in output a best-match 3D-DXA image volume and a variety of geometrical and bone mineral density parameters (Humbert et al., 2017). Finite-element calculation of bone strength based on DXA images have been validated against experimental results (Dall'Ara et al., 2016; Grassi et al., 2017) and shown to improve aBMD clinical classification of fracture and non-fracture cases (AUC $>0.8$ vs. AUC $<0.7$ ) (Wills et al., 2019). Yet, the repeatability of 3D-DXA images for assessing exercise-induced hip BMD and strength changes is unclear.

The aim of this study was 1) to quantify the repeatability of vBMD and femoral neck strength obtained from 3D-DXA images and 2) to determine vBMD and femoral neck strength changes in an exercise group of reference in relation to corresponding repeatability values. To this purpose, we developed a method for assessing changes in BMD and femoral neck strength from 3D-DXA images. The repeatability of the 3D-DXA method was quantified using singleday repeated images in a cohort of healthy women for which no BMD and femoral neck strength changes are to be expected. Spatial BMD and femoral neck strength changes following the exercise intervention by Harding and co-workers (Harding et al., 2020a), used as reference, 
METHODS

$3 D-D X A$ data

Two sets of DXA images were obtained using the same DXA machine (Medix DR,

Medilink, France). The first set of images included pairs of same-day repeated DXA scans of the hip for ten healthy female participants ( $54 \pm 7.1$ years). The second set of images included pre- and post-exercise intervention DXA scans of the proximal femur of 27 male participants $(64.9 \pm 8.6$ years $)$ taken from an earlier eight-month, semi-randomized controlled exercise intervention trial (Harding et al., 2017). The participants analysed in the current report were from the high-intensity resistance and impact training (HiRIT) group. The HiRIT program incorporated multi-joint, compound movement, high-intensity progressive resistance training and high-impact jumping exercises and was shown to promote a moderate aBMD response in the proximal femur (Harding et al., 2020a; Harding et al., 2020b).

Femoral neck strength calculation

The 3D-DXA images calibrated to equivalent bone mineral content levels were obtained with 3D-SHAPER software (v.2, Galgo Medical, Barcelona, Spain) (Humbert et al., 2017). Briefly, the software uses a 3D statistical shape and appearance model (SSAM) of the proximal femur built from a database of QCT scans. The database included 111 Caucasian subjects (30 male and 81 female) with a mean age of $56 \pm 12$ years and no signs of skeletal disease other than osteoporosis. The software delivers a 3D volume of images of the proximal femur from the planar DXA image.

The proximal femur surface geometry for each 3D-DXA image was retrieved through threshold-based segmentation and meshed with triangular elements. The triangulated meshes underwent 5 iterations of smoothing using curvature flow smoothing (Desbrun et al., 1999) 
to-point distance error of $0.09 \pm 0.38 \mathrm{~mm}$ between the retrieved and smoothed geometries across all repeatability participants. Geometrical errors of this size were found to cause $<10 \%$ error in the maximum and minimum principal strains (Taddei et al., 2006).

A template surface mesh was generated from the 20 proximal femoral geometries used in the repeatability set of images, by first morphing one femur surface mesh to all 20 femurs and then calculating the mean shape. The template mesh was characterised by a triangular surface mesh (33,954 elements). A smoothing algorithm was applied to the surface mesh for regularity so that $90 \%$ of the elements had a Jacobian $>0.8$ and minimum edge sizes of $1.25-$ $1.75 \mathrm{~mm}$. A template volume mesh of linear tetrahedral elements $(205,233$ elements $)$ was generated from the surface mesh using an advancing front algorithm (Hypermesh 14.0, Altair Engineering Inc., Troy, USA).

The template was morphed over the shape of each bone in the dataset in multiple steps. Firstly, the template surface mesh was rigidly registered to the target surface mesh by aligning the principal axes of inertia of the template and the target. Secondly, the rigid registration was optimized with a rigid iterative closest point (ICP) algorithm so that rotation, translation, and scaling of the template vertices were iteratively calculated as solutions to a weighted least squares minimisation problem by Singular Value Decomposition (SVD). The algorithm converged when the root mean squared error (RMSE) between the previous and current template vertices dropped below a threshold value $\left(<10^{-5} \mathrm{~mm}\right)$. Third, an ICP based non-rigid registration algorithm, modelled as a sum of Gaussian Radial Basis Functions (G-RBF) with 10 iterations, was applied to locally deform the template surface mesh to match the geometry of the target surface mesh (Audenaert et al., 2019). Finally, a custom finite element solution (MATLAB 2018b, The MathWorks Inc., MA, USA) was implemented in which the displacements at the nodes on the surface of the template volume mesh were imposed to equal 
those calculated while registering the surface meshes. This process delivered morphed volume meshes with node numbering and connectivity correspondence.

Bone was assumed to be linear-elastic and have inhomogeneous isotropic material

100 properties. The material properties were mapped to the morphed template volume mesh from the 3D-DXA image. A calibration correction was applied to the radiological density obtained in the 3D-SHAPER modelling process to estimate ash density (Schileo et al., 2008a). An empirical relationship was then used to convert the ash density $\left(\mathrm{g} / \mathrm{cm}^{3}\right)$ to elastic modulus (MPa) (Morgan et al., 2003) with an ash-to-wet density correction applied (Schileo et al., 2008a):

$$
\mathrm{E}=14644 \times \rho^{1.49}
$$

107 The elastic modulus distribution was integrated over the element volume using Gaussian 108 quadrature with an in-house custom code (MATLAB 2018b, The MathWorks Inc., MA, USA) delivering the element modulus used for FEA. The Poisson's ratio was set to 0.3.

111 the proximal femoral shaft above and below the lesser trochanter. The line joining the centre 112 points of the node rings defined the Z-axis. The Z-X plane was defined by establishing a 113 temporary axis between the centre point above the lesser trochanter and the centre of the 114 femoral head. The Y-axis was defined as the cross-product of the Z-axis and temporary axis in the Z-X plane. The $\mathrm{X}$-axis was defined as the cross-product of the Z-axis and Y-axis. Two sets of boundary conditions were used, one representing a static single-leg stance

117 load configuration and the other representing a fall on the side (Figure 2). The single-leg stance 118 configuration was simulated by constraining the distal end of the femur. A nominal load of 100

$119 \mathrm{~N}$ lying in the frontal plane and passing through the femoral head centre at $8^{\circ}$ abduction from 120 the diaphysis was applied (Cristofolini et al., 2007). The force was distributed over a $5 \mathrm{~mm}$ 
121 diameter node patch on the superior femoral head surface to minimise numerical artefacts. To account for variation in load direction, nine simulations were run for the single-leg stance loading configuration with the load direction varied by $\pm 5^{\circ}$, and $\pm 10^{\circ}$ from the standard load

124 case in the coronal and sagittal planes.

Similarly, the sideways fall loading configuration was simulated with a nominal load of $100 \mathrm{~N}$ distributed over a $5 \mathrm{~mm}$ diameter node patch on the medial side of the femoral head and pointing laterally with $0^{\circ}$ rotation in the coronal and axial planes of the local co-ordinate system. The loading direction for sideways fall was varied in the coronal and axial planes by \pm $5^{\circ}$ and $\pm 10^{\circ}$ from the standard load case. The distal extremity of the femur was free to rotate

130 about the anterior-posterior axis and the surface of the greater trochanter was constrained in the medial-lateral direction. All simulations were performed in ANSYS (19.1, ANSYS Inc., PA, USA).

The femoral neck strength was calculated for each femur and loading configuration using a maximum principal strain criterion (Schileo et al., 2014; Schileo et al., 2008b). The maximum and minimum principal bone strains were recorded for each element in a region of interest on the external surface of the femoral neck (Appendix 1). The region of interest was set below the head-neck junction to minimize the effect of the strain concentration caused by

138 the load application on the results. The risk of fracture (RF) was calculated as the ratio between

139 the maximum principal and minimum principal strain ( $\left.\varepsilon_{\mathrm{MAX}}\right)$ and the asymmetric elastic limit 140 values of bone in compression $\left(\varepsilon_{\mathrm{LIM}}=10,000 \mu \varepsilon\right)$ and tension $\left(\varepsilon_{\mathrm{LIM}}=7,000 \mu \varepsilon\right)$ (Bayraktar et 141 al., 2004). Bone strength (Fs) was determined as the nominal load applied (100 N) over the 142 maximum RF:

$$
\mathrm{Fs}=\mathrm{F}_{\text {nominal }} / \mathrm{RF}_{\max }
$$


147 in the total hip region, vBMD distribution, and strength. The difference in bone outcomes

148 between repeated scans was calculated and expressed as a percentage of the corresponding

149 mean value. Femoral neck strength differences were calculated for both the nominal single-leg stance and sideways fall configurations and the corresponding eight perturbations of loading

151 direction. The effect of geometric and BMD differences and that of perturbations of the loading

152 direction were analysed by calculating the standard deviation of the pooled results obtained for

153 all 10 participants and all the nine loading orientations, respectively.

Bone and strength changes in the exercise group were analysed using linear regression

(MATLAB, The MathWorks Inc., Natick, USA) for both the single-leg-stance and the side fall loading using the 64 different parameters provided by the 3D-SHAPER software describing bone geometry and quality (Appendix 2). The significance level for regression was set at 0.05 .

158 The spatial BMD changes in the exercise group and the repeatability group were compared by displaying the element-by-element mean BMD change in the exercise group with respect to the mean BMD change in the repeatability group. We also examined the spatial BMD and strength changes in individual participants who represented the extreme cases of total hip vBMD and femoral neck strength changes in the exercise group to provide an insight into the validity limits of 3D-DXA analyses.

\section{RESULTS}

The total hip BMD difference in repeated images cumulatively averaged over 6 or more participants was consistently below $0.5 \%$ and the standard deviation was consistently within

167 the $2.5-2.7 \%$ range (Table 1). Local element-by-element BMD differences reached $0.06 \pm$ $1680.12 \mathrm{~g} / \mathrm{cm}^{3}(30 \pm 50 \%)$ in the greater trochanter and calcar regions (Figure 3). The peak strain 
169 in single-leg stance occurred within the region of interest (i.e. superior surface of the femoral

170 neck) except for participants 3, 4, 6, and where it occurred on posterior aspect of the femur

171 near the intertrochanteric ridge. By grouping the femoral neck strength differences obtained

172 for each participant, the average standard deviation was $13 \%$ for sideways fall and $19 \%$ for

173 single-leg stance loading, while pooling the strength differences obtained for each load 174 orientation, the average standard deviation was $3 \%$ for sideways fall and $11 \%$ for single-leg 175 stance loading (Table 2).

In the exercise group, femoral neck strength improved by $6 \pm 19 \%$ following exercise 177 under single-leg stance loading and by $1 \pm 18 \%$ under side fall loading (Appendix 3). Femoral 178 neck strength changes under a single-leg stance loading were weakly, or moderately, associated with positive changes in 13 of the 64 parameters analysed including both geometric and bone quality parameters (Table 3). The total hip BMD changes were $1.3 \pm 2.8 \%$ in the exercise group. The highest coefficient of determination between bone and femoral neck strength changes $\left(\mathrm{R}^{2}=0.31\right)$ was found for the femoral neck bone mineral content $(\mathrm{BMC})$ for the single-

183 leg stance loading (Table 3$)$. There was either no association or very weak association $\left(\mathrm{R}^{2}<\right.$ 1840.1 ) between changes of every bone parameter analysed and changes of femoral neck strength under side fall loading. changes were comparable across the entire bone volume in terms of mean BMD and standard deviation (Figure 3 and Figure 4). Nevertheless, the exercise group displayed a mean bone accrual exceeding corresponding repeated values in the femoral head and cortical regions and a mean bone decrease exceeding corresponding repeated values predominantly in the sub-

191 cervical and the calcar regions (Figure 5). 

and $-7 \%$ change of femoral neck strength under single-leg and sideways fall loading. The highest negative change (-5.1\%) largely occurred in the calcar region causing an $8 \%$ and $-8 \%$ change of femoral neck strength under single-leg stance and sideways fall loading, respectively (Figure 6). Furthermore, the highest positive change of femoral neck strength (63\%) occurred under single-leg stance loading in response to a $1.3 \%$ increase of the total hip vBMD while under sideways fall loading the femoral neck strength change was $-0.2 \%$ (Figure 7 ).

DISCUSSION

The aim of the present study was to compare the repeatability of BMD and femoral neck strength estimates to corresponding changes caused by exercise using a common 3DDXA procedure. The 3D-DXA procedure analysed provided repeatable changes of typical BMD parameters calculated over large bone portions and strength averaged over 6 participants or more to enable a mean error below $0.5 \%$. Changes of strength were load-dependent and were about one order of magnitude higher than corresponding BMD changes. Local (element-byelement) BMD values displayed a much higher dispersion than common vBMD parameters

207 similar in the repeatability and the exercise group. Therefore, the 3D-DXA technology analysed can be used to determine the main effect of an exercise intervention on commonly used vBMD parameters and strength. However, the validity of BMD and femoral neck strength changes on a case-by-case base should be considered with caution.

212 consistently below $0.5 \%$ and $5 \%$ when six to ten participants were considered, providing the minimal effect size that can be detected using the 3D-DXA procedure analysed. Larger cohorts

214 may enable detection of a smaller effect size. Interestingly, the variation of the element-by215 element $\mathrm{BMD}(\mathrm{SD}=50 \%)$ was much higher than that in commonly used geometrical and 216 vBMD parameters analysed $(\mathrm{SD}=2.5-2.7 \%)$, hence contributing to the variance in femoral 
neck strength, which was found to be affected by geometrical and BMD differences $(13 \%-$ 218 19\%) and load direction $(3 \%-11 \%)$. This information is important for understanding the 219 variations observed in 3D-DXA analyses.

For the exercise group, femoral neck strength increased by $6 \%$ and $1 \%$ under singleleg stance and sideways fall loading, showing a load-dependent strength response above and below the respective repeatability values. Only $20 \%$ (13 of 64$)$ of the geometrical and BMD parameters analysed here were weakly, or moderately, correlated to femoral neck strength changes indicating local variations are important determinants of bone strength. This finding supports the notion that inferring strength from BMD measurement may lack sensitivity and can be misleading (Turner and Robling, 2005). However, the exercise group displayed a larger but comparable variation of BMD and femoral neck strength changes (SD: $2.8 \%$ and $18-19 \%$ ) to those in the repeated images (SD: $2.5-2.7 \%$ and $13-19 \%$ ), suggesting that the 3D-DXA procedure analysed here should be used with caution on a case-by-case basis.

Although extreme cases in the exercise group analysed here may not well represent BMD and strength changes in the participants, the extreme cases can provide insights into understanding of the potential of exercise for promoting bone strength. For example, 1.3\% increase in total hip vBMD caused a $63 \%$ femoral neck strength increase under a single-leg stance loading in one participant. Therefore, it appears that exercise can have a remarkable effect on strength despite inducing a relatively modest BMD response. Yet, the optimal exercise type and amount for optimal hip strength in each participant remains to be determined

237 (Martelli et al., 2020).

The present results compare well with earlier findings. The larger changes of femoral neck strength $(6 \%)$ over that of BMD $(1.3 \%)$ is in agreement with the notion that exercise, in contrast to drug therapies alone that act systemically, best promote bone strength by causing 
241 local bone changes (Warden et al., 2020) at the highest possible distance from the neutral axis

242 thereby best promoting strength (Oden et al., 1999). The load-dependent response of femoral

243 neck strength to exercise can be attributed to the exercise type which depends on hip load

244 orientation and intensity resulting from an exercise-specific muscle contraction (Martelli et al.,

245 2014; Martelli et al., 2017) causing a spatially heterogeneous bone mechanical stimulus and 246 response.

There were limitations in the present study. The use of a single DXA device has enabled to quantify the repeatability of the 3D-DXA procedure alone. However, the use of multiple devices may lead to increased dispersion of the data. Also, the assumption of linear elastic and brittle fracture behaviour affected the calculation of femoral neck strength. However, models implementing linear and non-linear elastic bone behaviour similarly explain $89 \%$ and $80-85 \%$ of the variance of the fracture load (Dall'Ara et al., 2013), hence providing confidence on the use of linear models in the present study. Nevertheless, bone strain and strength calculation based on 3D-DXA images was found less accurate than corresponding calculation based on CT images (Grassi et al., 2017) due to repeatability errors in the 3D-DXA reconstruction process. Addressing this limitation may require improving, eventually via dedicated validation studies, the repeatability of the participant position during imaging, the search algorithm for extracting the 3D volume from the SSAM model and/or the SSAM model itself, and improving the representativeness of the training set.

In conclusion, this study quantified the repeatability of a specific 3D-DXA procedure

261 for the assessment of volumetric BMD and femoral neck strength and compared the 262 repeatability analysis with corresponding values following a selected exercise intervention of 263 reference. As such, the present findings can inform the use of 3D-DXA technologies for the 264 analysis and the design of exercise therapy for promoting hip health. The present results support 265 the use of the 3D-DXA technology analysed for studying the main effect of exercise 
interventions. However, the use of the 3D-DXA technology for the analysis of bone density

267 and strength changes caused by exercise in individual participants should be considered with

268 caution.

\section{ACKNOWLEDGEMENTS}

Australian Research Council (DP180103146; FT180100338).

\section{REFERENCES}

Abrahamsen, B., Van Staa, T., Ariely, R., Olson, M., Cooper, C., 2009. Excess mortality following hip fracture: A systematic epidemiological review. Osteoporosis International 20, 1633-1650.

Audenaert, E.A., Van Houcke, J., Almeida, D.F., Paelinck, L., Peiffer, M., Steenackers, G., Vandermeulen, D., 2019. Cascaded statistical shape model based segmentation of the full lower limb in CT. Computer Methods in Biomechanics and Biomedical Engineering 22, 644-657.

280 Bayraktar, H.H., Morgan, E.F., Niebur, G.L., Morris, G.E., Wong, E.K., Keaveny, T.M., 2004. tissue. Journal of Biomechanics 37, 27-35.

283 Beck, B.R., Daly, R.M., Singh, M.A.F., Taaffe, D.R., 2017. Exercise and Sports Science

Australia (ESSA) position statement on exercise prescription for the prevention and management of osteoporosis. Journal of Science and Medicine in Sport 20, 438-445.

286 Cheng, X.G., Lowet, G., Boonen, S., Nicholson, P.H.F., Brys, P., Nijs, J., Dequeker, J., 1997. Assessment of the strength of proximal femur in vitro: Relationship to femoral bone mineral density and femoral geometry. Bone 20, 213-218.

Cristofolini, L., Juszczyk, M., Martelli, S., Taddei, F., Viceconti, M., 2007. In vitro replication of spontaneous fractures of the proximal human femur. Journal of Biomechanics 40, 28372845 .

292 Dall'Ara, E., Luisier, B., Schmidt, R., Kainberger, F., Zysset, P., Pahr, D., 2013. A nonlinear 293 QCT-based finite element model validation study for the human femur tested in two 294 configurations in vitro. Bone 52, 27-38.

295 Dall'Ara, E., Eastell, R., Viceconti, M., Pahr, D., Yang, L., 2016. Experimental validation of 296 DXA-based finite element models for prediction of femoral strength. Journal of the Mechanical 297 Behavior of Biomedical Materials 63, 17-25. 

using diffusion and curvature flow. In Proceedings of the 26th Annual Conference on Computer Graphics and Interactive Techniques, SIGGRAPH 1999.

Falcinelli, C., Schileo, E., Balistreri, L., Baruffaldi, F., Bordini, B., Viceconti, M., Albisinni, U., Ceccarelli, F., Milandri, L., Toni, A., Taddei, F., 2014. Multiple loading conditions analysis can improve the association between finite element bone strength estimates and proximal femur fractures: A preliminary study in elderly women. Bone 67, 71-80.

Grassi, L., Väänänen, S.P., Ristinmaa, M., Jurvelin, J.S., Isaksson, H., 2017. Prediction of femoral strength using 3D finite element models reconstructed from DXA images: validation against experiments. Biomechanics and modeling in mechanobiology 16, 989-1000.

Harding, A.T., Weeks, B.K., Lambert, C., Watson, S.L., Weis, L.J., Beck, B.R., 2020a. A Comparison of Bone-Targeted Exercise Strategies to Reduce Fracture Risk in Middle-Aged and Older Men with Osteopenia and Osteoporosis: LIFTMOR-M Semi-Randomized Controlled Trial. Journal of Bone and Mineral Research.

312 Harding, A.T., Weeks, B.K., Lambert, C., Watson, S.L., Weis, L.J., Beck, B.R., 2020b. Effects of supervised high-intensity resistance and impact training or machine-based isometric training 314 on regional bone geometry and strength in middle-aged and older men with low bone mass: The LIFTMOR-M semi-randomised controlled trial. Bone 136, 115362.

316 Harding, A.T., Weeks, B.K., Watson, S.L., Beck, B.R., 2017. The LIFTMOR-M (Lifting 317 Intervention for Training Muscle and Osteoporosis Rehabilitation for Men) trial: Protocol for 318 a semirandomised controlled trial of supervised targeted exercise to reduce risk of osteoporotic 319 fracture in older men with low bone mass. BMJ Open 7.

320 Humbert, L., Martelli, Y., Fonolla, R., Steghofer, M., Di Gregorio, S., Malouf, J., Romera, J., Barquero, L.M.D.R., 2017. 3D-DXA: Assessing the Femoral Shape, the Trabecular Macrostructure and the Cortex in 3D from DXA images. IEEE Transactions on Medical Imaging 36, 27-39.

Johnell, O., Kanis, J.A., 2006. An estimate of the worldwide prevalence and disability associated with osteoporotic fractures. Osteoporosis International 17, 1726-1733.

Lang, T.F., Saeed, I.H., Streeper, T., Carballido-Gamio, J., Harnish, R.J., Frassetto, L.A., Lee, Cavanagh, P.R., 2014. Spatial heterogeneity in the response of the proximal femur to two lower-body resistance exercise regimens. Journal of Bone and Mineral Research 29, 13371345 .

Martelli, S., Beck, B., Saxby, D., Lloyd, D., Pivonka, P., Taylor, M., 2020. Modelling Human Locomotion to Inform Exercise Prescription for Osteoporosis. Current Osteoporosis Reports $18,301-311$.

334 Martelli, S., Kersh, M.E., Schache, A.G., Pandy, M.G., 2014. Strain energy in the femoral neck during exercise. Journal of Biomechanics 47, 1784-1791.

336 Martelli, S., Mokhtarzadeh, H., Pivonka, P., Ebeling, P.R., 2017. The Femoral Neck 337 Mechanoresponse to Hip Extensors Exercise: A Case Study. Journal of Osteoporosis 2017. 
Morgan, E.F., Bayraktar, H.H., Keaveny, T.M., 2003. Trabecular bone modulus-density relationships depend on anatomic site. Journal of Biomechanics 36, 897-904.

Oden, Z.M., Selvitelli, D.M., Bouxsein, M.L., 1999. Effect of local density changes on the failure load of the proximal femur. Journal of Orthopaedic Research 17, 661-667.

Qasim, M., Farinella, G., Zhang, J., Li, X., Yang, L., Eastell, R., Viceconti, M., 2016. Patientspecific finite element estimated femur strength as a predictor of the risk of hip fracture: the effect of methodological determinants. Osteoporosis International 27, 2815-2822.

Rubin, C.T., Lanyon, L.E., 1985. Regulation of bone mass by mechanical strain magnitude. Calcified Tissue International 37, 411-417.

Schileo, E., Balistreri, L., Grassi, L., Cristofolini, L., Taddei, F., 2014. To what extent can linear finite element models of human femora predict failure under stance and fall loading configurations? Journal of Biomechanics 47, 3531-3538.

Schileo, E., Dall'Ara, E., Taddei, F., Malandrino, A., Schotkamp, T., Baleani, M., Viceconti, M., 2008a. An accurate estimation of bone density improves the accuracy of subject-specific finite element models. Journal of Biomechanics 41, 2483-2491.

Schileo, E., Taddei, F., Cristofolini, L., Viceconti, M., 2008b. Subject-specific finite element models implementing a maximum principal strain criterion are able to estimate failure risk and fracture location on human femurs tested in vitro. Journal of Biomechanics 41, 356-367.

Taddei, F., Martelli, S., Reggiani, B., Cristofolini, L., Viceconti, M., 2006. Finite-element modeling of bones from CT data: Sensitivity to geometry and material uncertainties. IEEE Transactions on Biomedical Engineering 53, 2194-2200.

Taddei, F., Palmadori, I., Taylor, W.R., Heller, M.O., Bordini, B., Toni, A., Schileo, E., 2014. European Society of Biomechanics S.M. Perren Award 2014: Safety factor of the proximal femur during gait: A population-based finite element study. Journal of Biomechanics 47, 34333440 .

Turner, C.H., Robling, A.G., 2005. Exercises for improving bone strength. British Journal of Sports Medicine 39, 188-189.

Warden, S.J., Carballido-Gamio, J., Weatherholt, A.M., Keyak, J.H., Yan, C., Kersh, M.E., Lang, T.F., Fuchs, R.K., 2020. Heterogeneous Spatial and Strength Adaptation of the Proximal Femur to Physical Activity: A Within-Subject Controlled Cross-Sectional Study. Journal of Bone and Mineral Research 35, 681-690.

Wills, C.R., Olivares, A.L., Tassani, S., Ceresa, M., Zimmer, V., Ballester, M.A.G., Del Río, L.M., Humbert, L., Noailly, J., 2019. 3D patient-specific finite element models of the proximal femur based on DXA towards the classification of fracture and non-fracture cases. Bone 121, 89-99.

Yang, L., Peel, N., Clowes, J.A., McCloskey, E.V., Eastell, R., 2009. Use of DXA-Based Structural Engineering Models of the Proximal Femur to Discriminate Hip Fracture. Journal of Bone and Mineral Research 24, 33-42. 


\section{D-DXA}

\section{Segment}

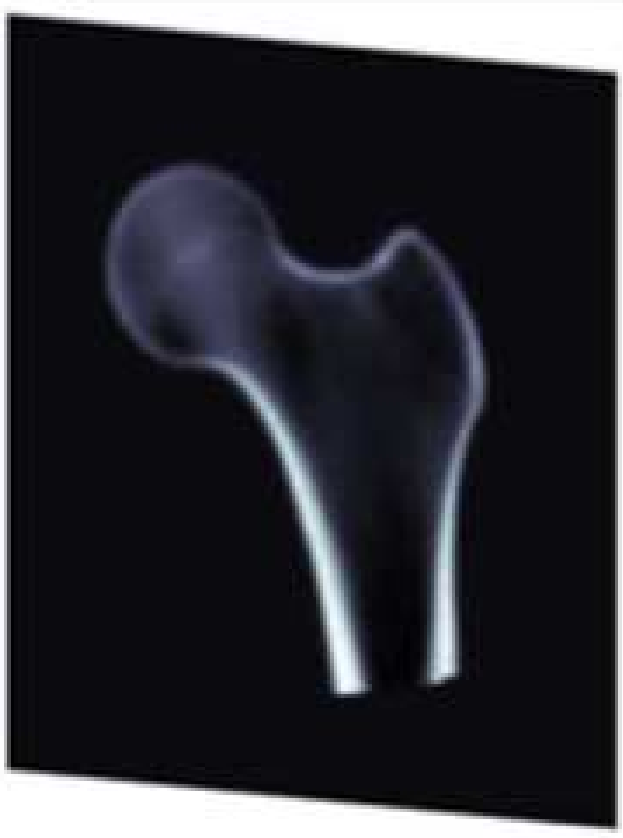

\section{D Femur Surface}
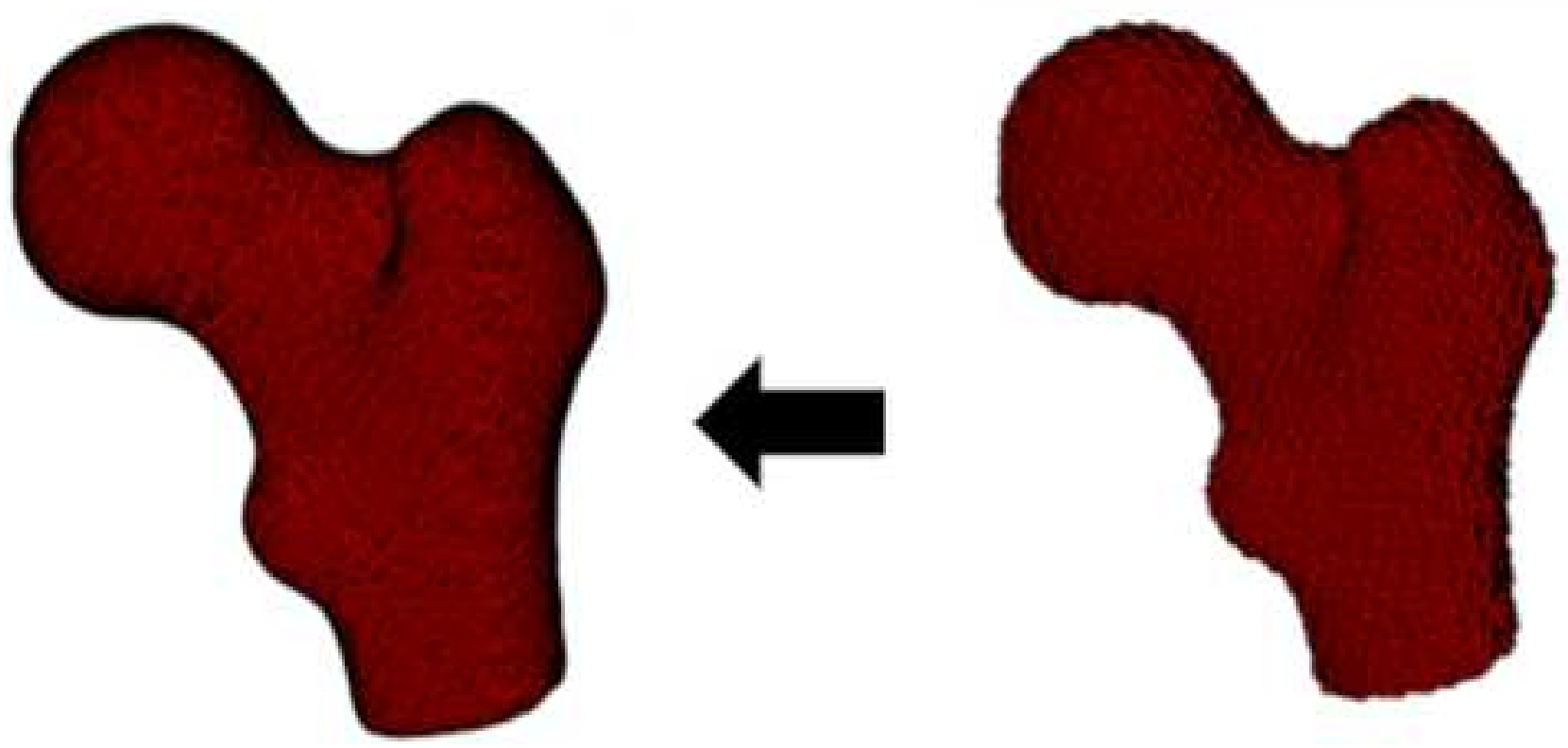

\section{Smoothing}
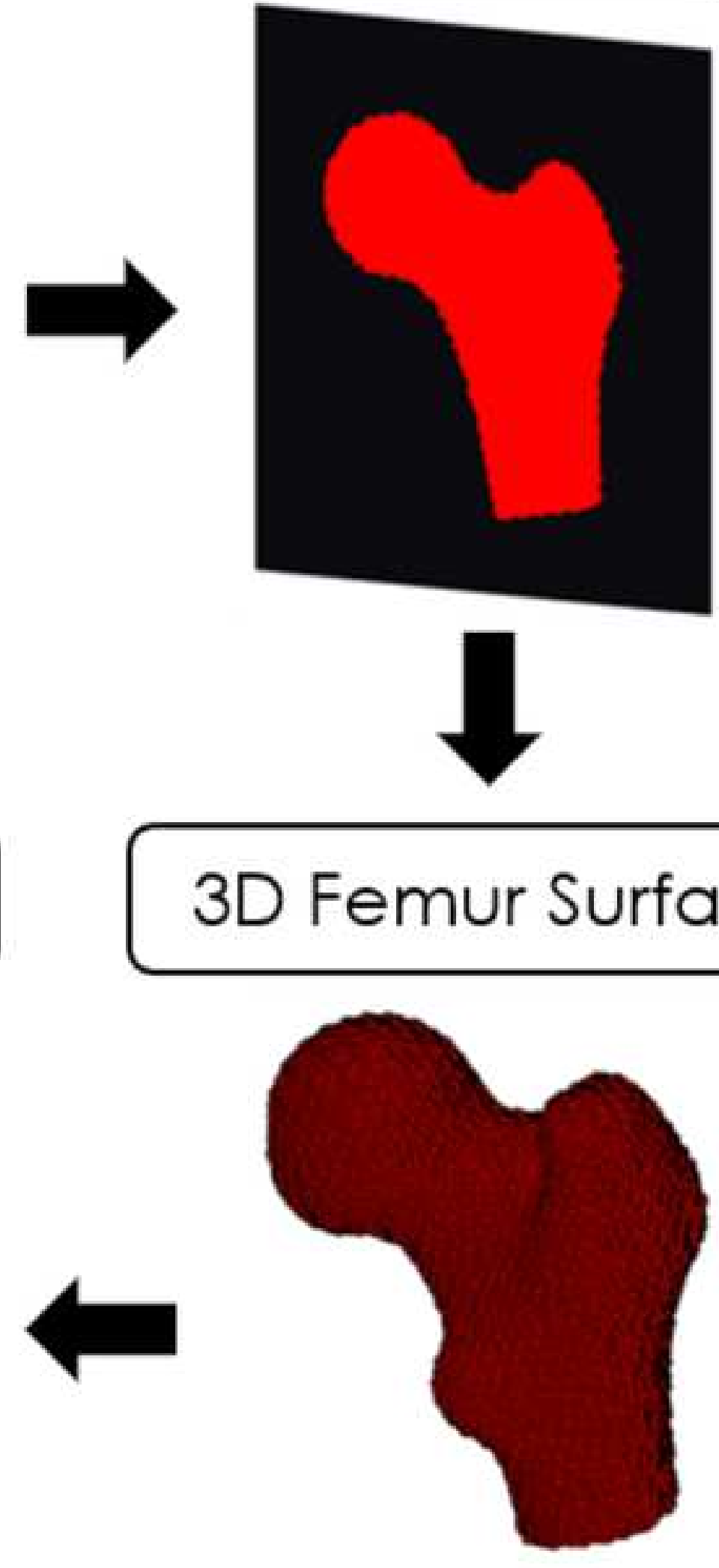

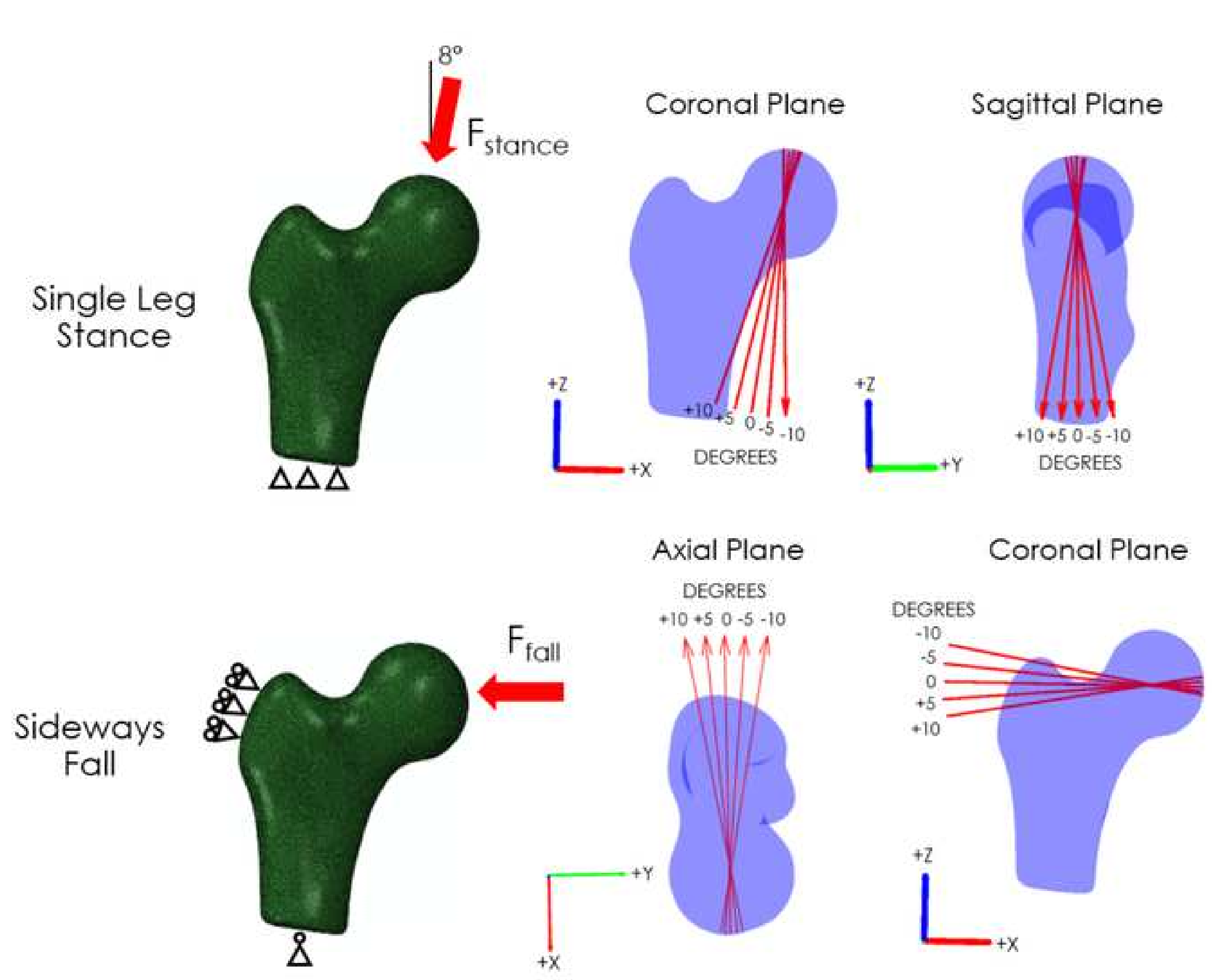
Mean

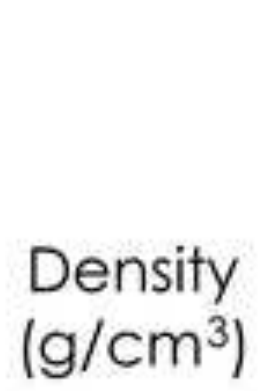

Density

(\%)

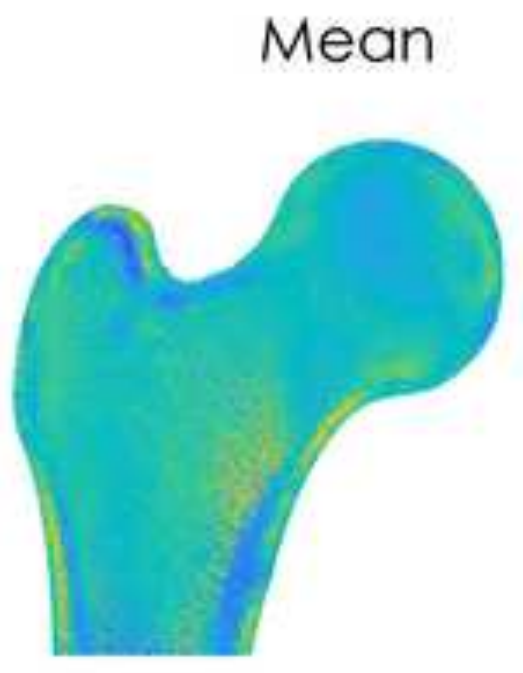

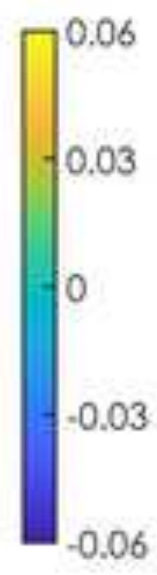

SD
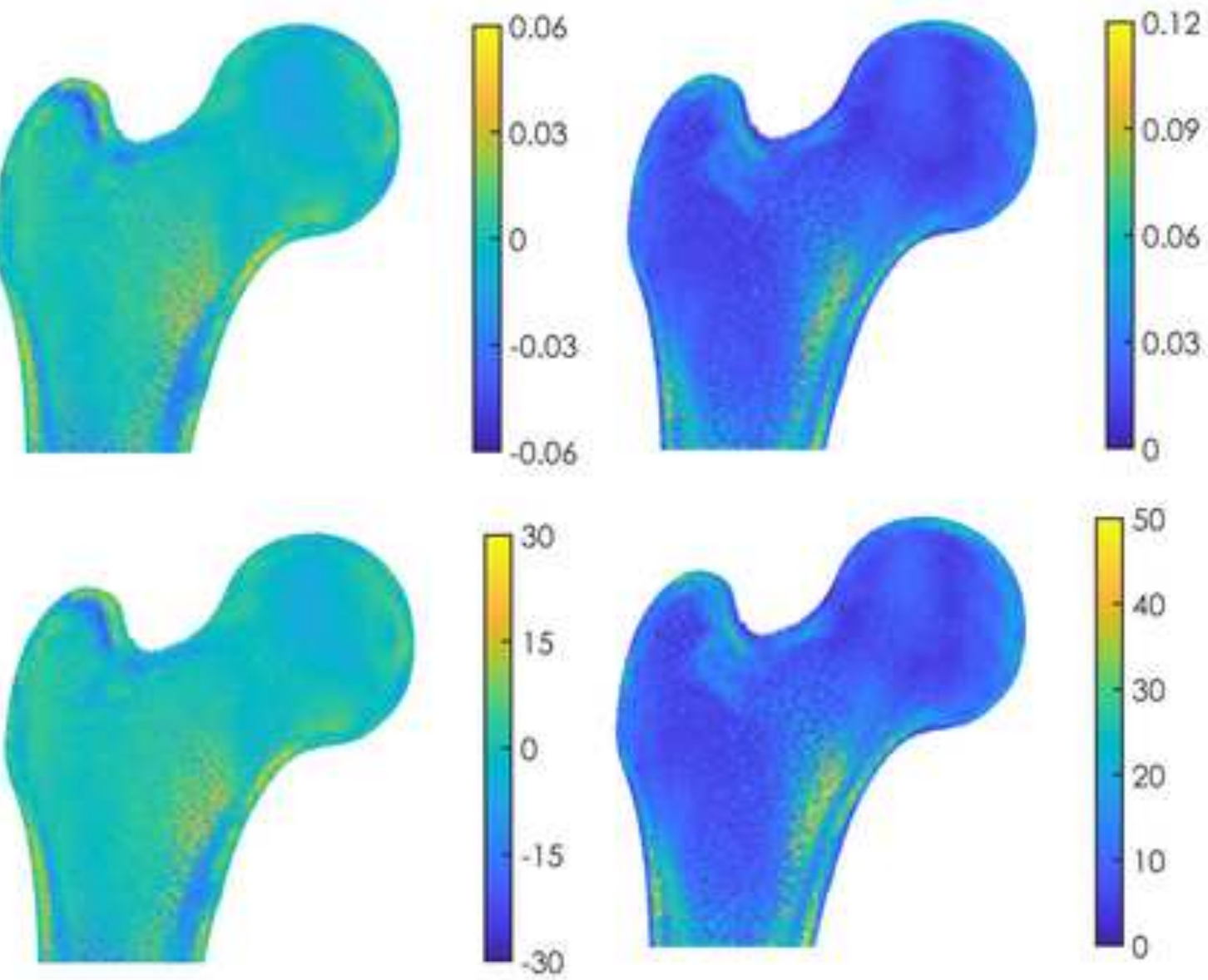

Single-leg Stance
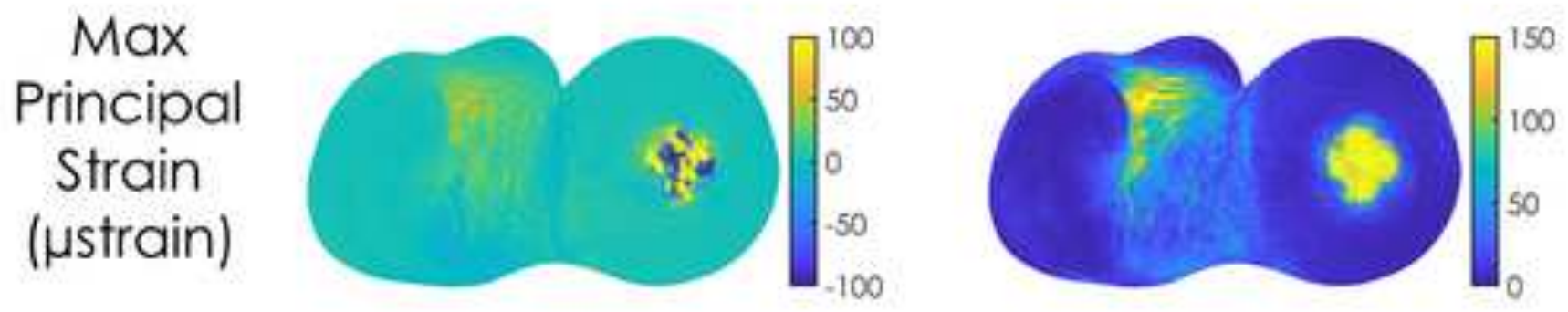

Sideways Fall
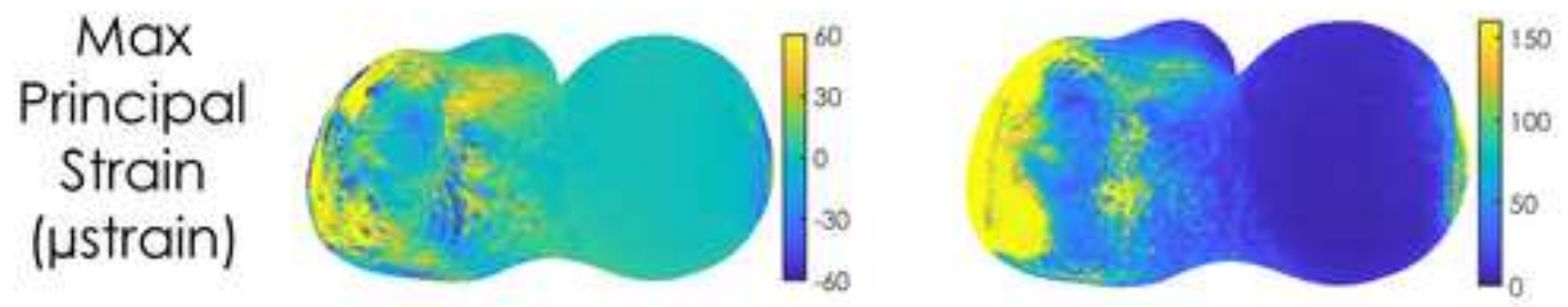


\section{Mean}

SD

Density $\left(\mathrm{g} / \mathrm{cm}^{3}\right)$
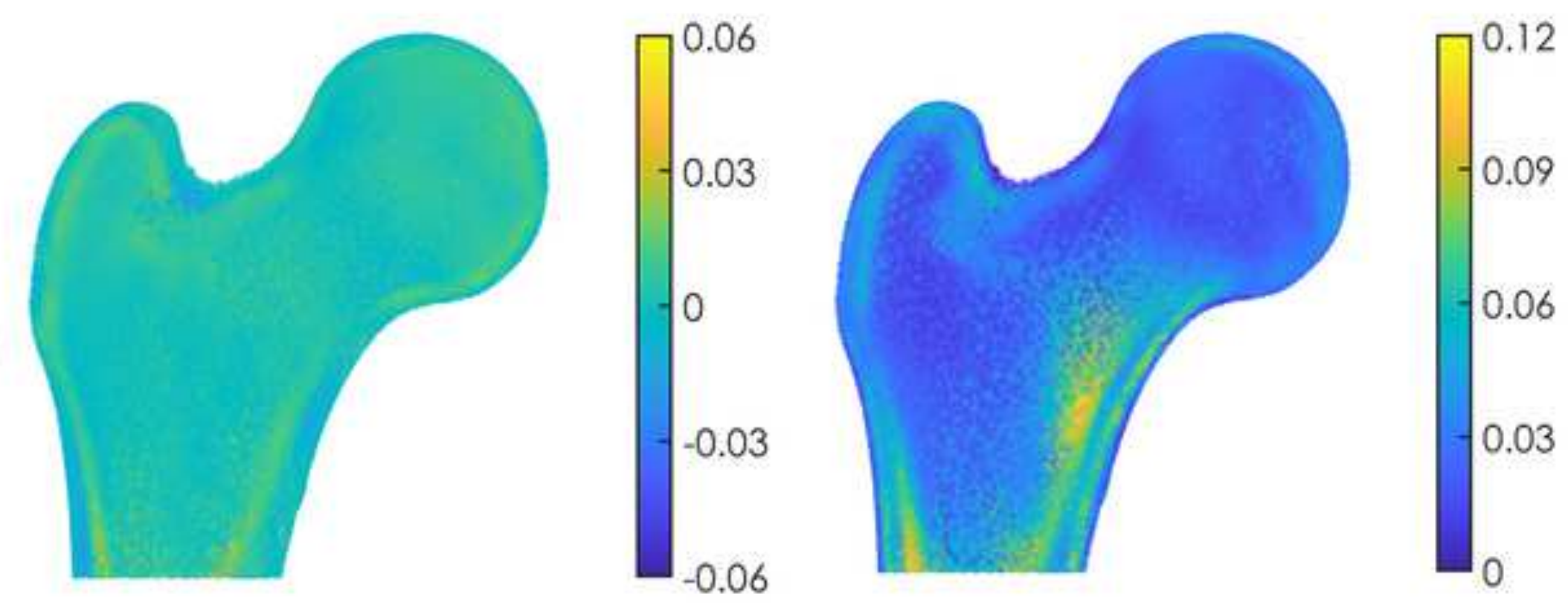

Density
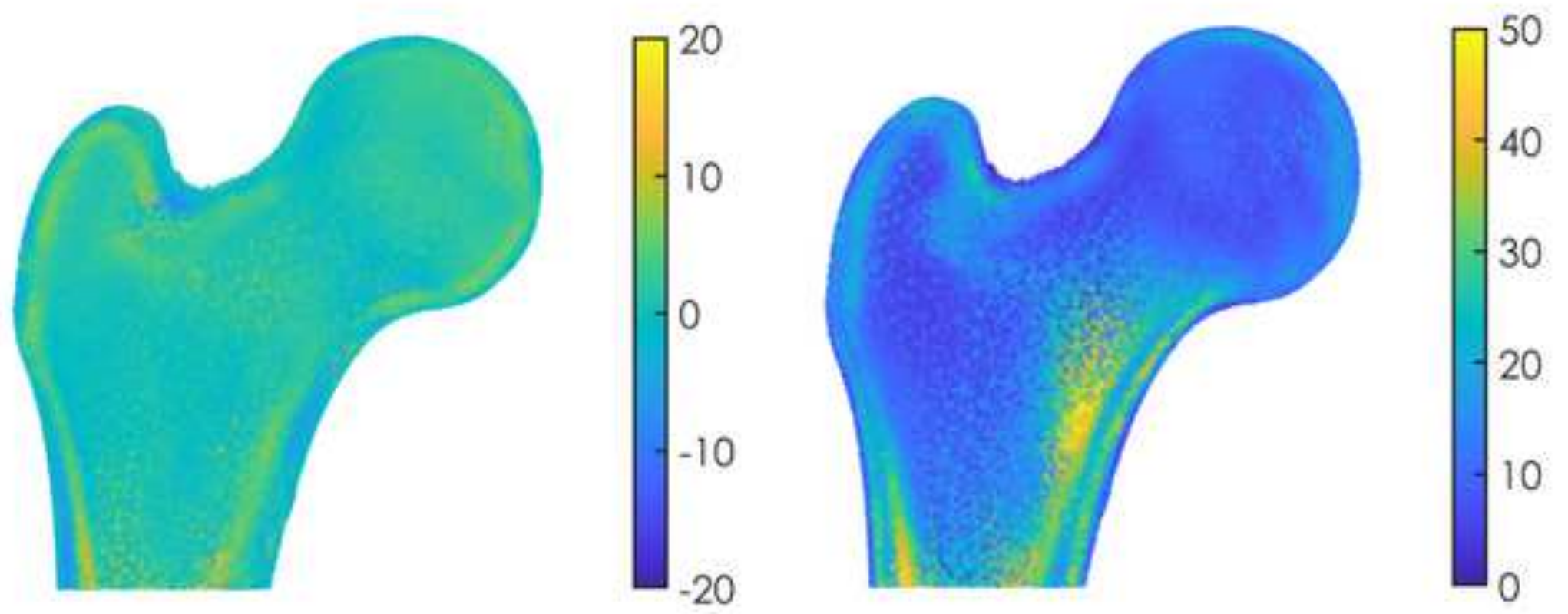


\section{Mean BMD Change - Exercise vs. Repeatability Group}

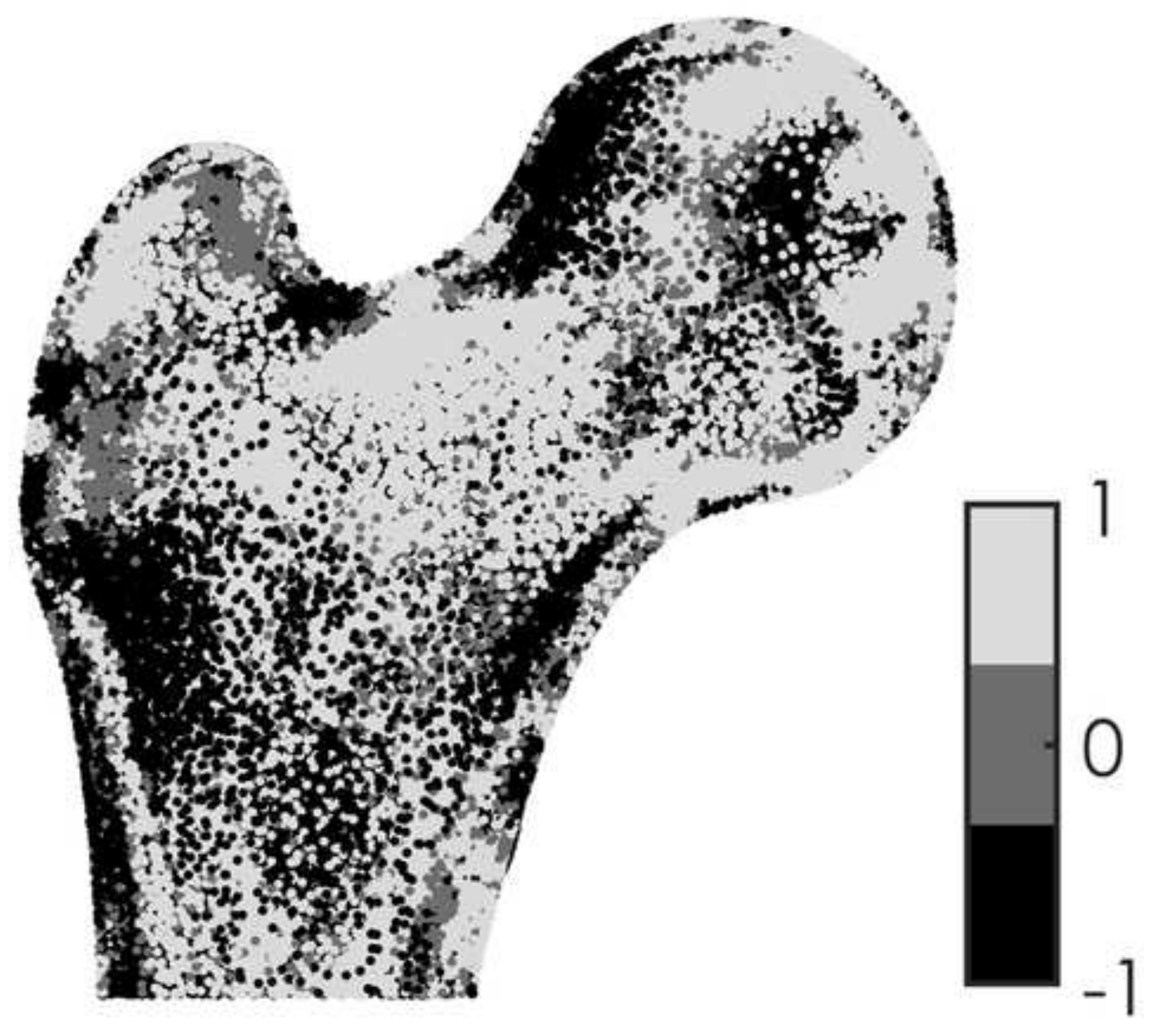


Min/Max Total Hip Integral vBMD Changes

Min Case

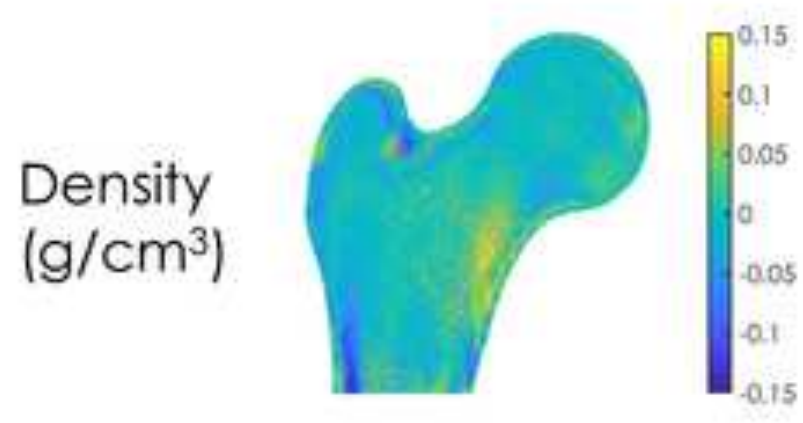

Single-leg Stance

Max

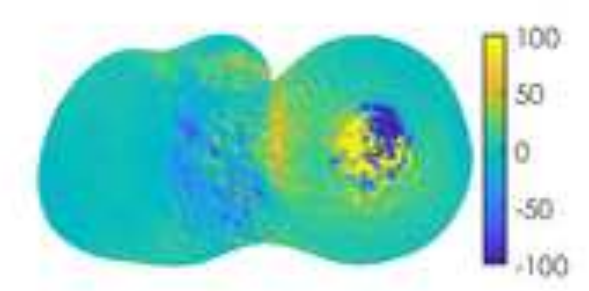

\section{Max Case}
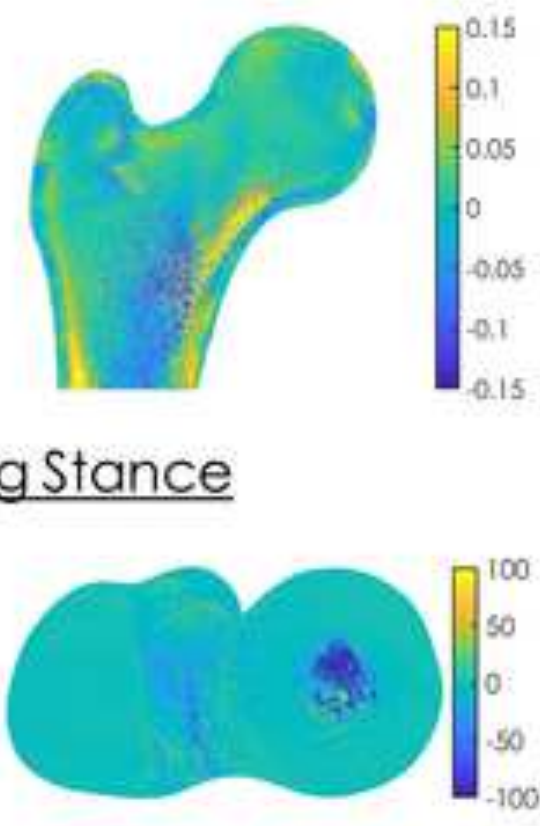

Sideways Fall

Strain

(ustrain)

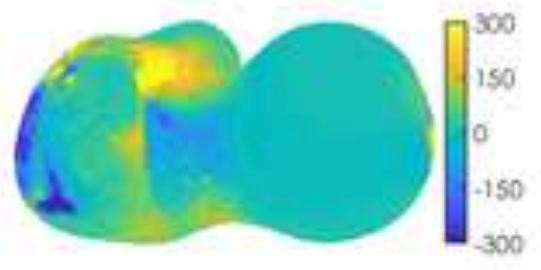

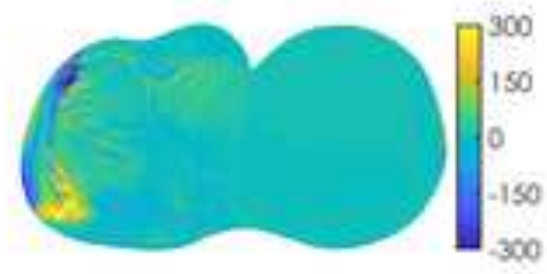

\section{Total Hip Integral $\mathrm{VBMD}\left(\mathrm{g} / \mathrm{cm}^{3}\right)$}

DIFF

INITIAL FINAL $\left(\mathrm{g} / \mathrm{cm}^{3}\right)$ DIFF (\%)

$\begin{array}{lllll}\text { MIN } & 0.239 & 0.227 & -0.012 & -5.1 \%\end{array}$

$\begin{array}{lllll}\text { MAX } & 0.303 & 0.322 & 0.019 & 6.4 \%\end{array}$

Fracture Load (N)

INITIAL FINAL DIFF (N) DIFF (\%)

$\begin{array}{lllll}\text { MIN } & 2603 & 2822 & 219 & 8 \%\end{array}$

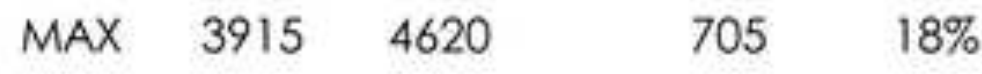

$\begin{array}{lrrrr} & \text { INITIAL } & \text { FINAL } & \text { DIFF (N) } & \text { DIFF (\%) } \\ \text { MIN } & 1280 & 1181 & -99 & -8 \% \\ \text { MAX } & 1384 & 1284 & -100 & -7 \%\end{array}$




\section{Min/Max Strength Changes}

Min Case

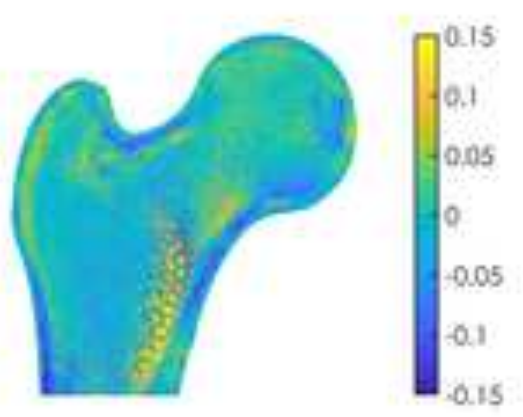

Max Case
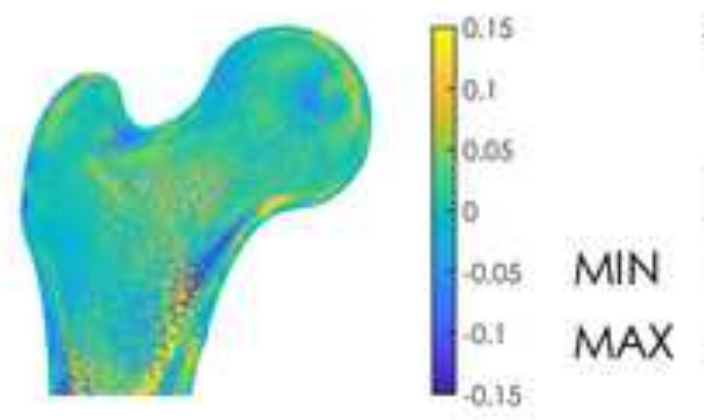

Total Hip Integral vBMD $\left(\mathrm{g} / \mathrm{cm}^{3}\right)$

DIFF

INITIAL FINAL $\left(\mathrm{g} / \mathrm{cm}^{3}\right)$ DIFF (\%)

$\begin{array}{llll}0.345 & 0.342 & -0.003 & -0.8 \%\end{array}$

$\begin{array}{llll}0.223 & 0.226 & 0.003 & 1.4 \%\end{array}$

Single-leg Stance

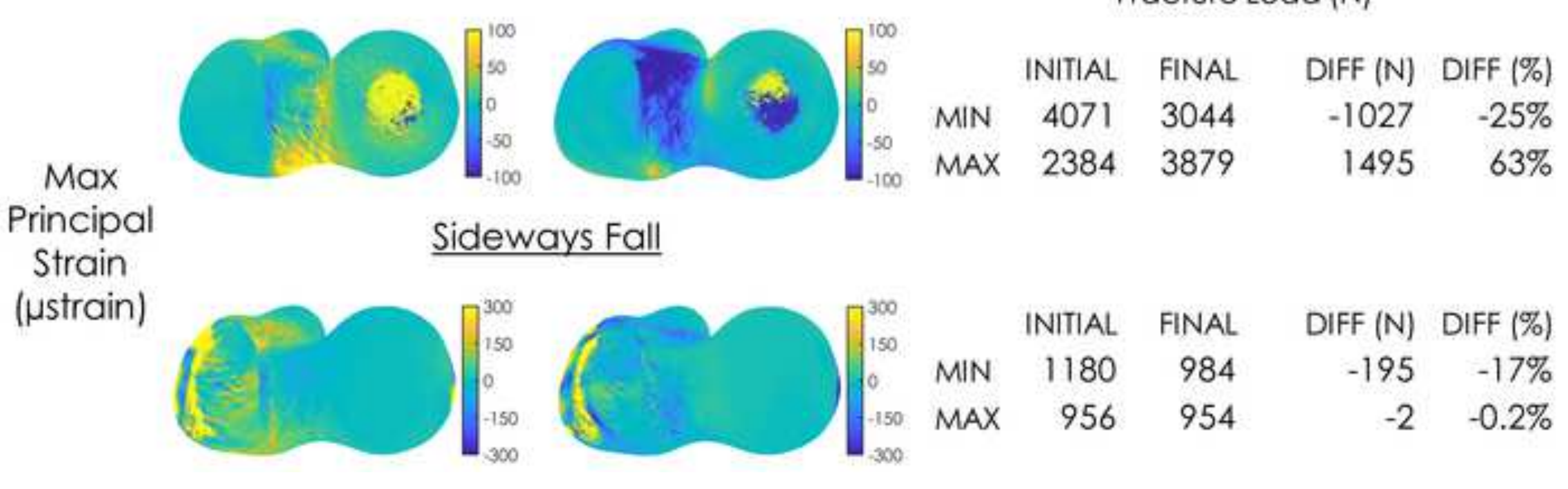


Table 1: Repeatability for the ten healthy female participants for the DXA-derived total hip integral vBMD.

\begin{tabular}{lrrrrrr}
\hline \multirow{2}{*}{ Participant } & \multicolumn{9}{c}{ Total Hip Integral vBMD } \\
& $\begin{array}{r}\text { SCAN1 } \\
\left(\mathrm{g} / \mathrm{cm}^{3}\right)\end{array}$ & $\begin{array}{r}\text { SCAN2 } \\
\left(\mathrm{g} / \mathrm{cm}^{3}\right)\end{array}$ & $\begin{array}{c}\text { DIFF } \\
\left(\mathrm{g} / \mathrm{cm}^{3}\right)\end{array}$ & $\begin{array}{r}\text { DIFF } \\
(\%)\end{array}$ & $\begin{array}{r}\text { Cumulative } \\
\text { Mean }\end{array}$ & $\begin{array}{r}\text { Cumulative } \\
001\end{array}$ \\
0.779 & 0.802 & 0.022 & 2.8 & 2.8 & - \\
002 & 0.754 & 0.753 & -0.001 & -0.1 & 1.3 & 2.1 \\
003 & 0.511 & 0.493 & -0.018 & -3.6 & -0.3 & 3.2 \\
004 & 0.598 & 0.591 & -0.007 & -1.2 & -0.5 & 2.6 \\
005 & 0.76 & 0.774 & 0.014 & 1.8 & -0.1 & 2.5 \\
006 & 0.609 & 0.632 & 0.023 & 3.7 & 0.6 & 2.7 \\
007 & 0.715 & 0.702 & -0.013 & -1.8 & 0.2 & 2.6 \\
008 & 0.482 & 0.492 & 0.01 & 2.1 & 0.5 & 2.5 \\
009 & 0.792 & 0.771 & -0.021 & -2.7 & 0.1 & 2.6 \\
010 & 0.842 & 0.87 & 0.029 & 3.4 & 0.4 & 2.7 \\
\hline
\end{tabular}


Table 2: Differences in femoral neck strength due to changes in geometry and to $\pm 5^{\circ}$ and \pm $10^{\circ}$ variation in loading direction. Sideways fall loading was varied in the superoinferior (SI) and the anteroposterior (AP) directions while single-leg stance was varied in the mediolateral (ML) and AP directions. Results presented are the percentage difference between the two scans at the given load case. The effect of geometrical and BMD changes was assessed by pooling the results obtained from each participant. The effect of load direction was assessed by pooling the results of all loading variations.

\begin{tabular}{|c|c|c|c|c|c|c|c|c|c|c|c|}
\hline \multicolumn{12}{|c|}{ Femoral Neck Strength Difference (\%) (SCAN2 - SCAN1) } \\
\hline \multirow[b]{3}{*}{ Participant } & \multirow[b]{3}{*}{ Standard } & \multirow[b]{3}{*}{$-10 S I$} & \multirow[b]{3}{*}{$-5 \mathrm{SI}$} & \multicolumn{3}{|c|}{ Sideways Fall } & \multirow[b]{3}{*}{$-5 \mathrm{AP}$} & \multirow[b]{3}{*}{$+5 \mathrm{AP}$} & \multirow[b]{3}{*}{$+10 \mathrm{AP}$} & & \\
\hline & & & & & & & & & & \multicolumn{2}{|c|}{ Average } \\
\hline & & & & $+5 \mathrm{SI}$ & +10 SI & $-10 \mathrm{AP}$ & & & & SD & SD \\
\hline 001 & -17 & -17 & -17 & -17 & -17 & -21 & -21 & -12 & -9 & 4 & \\
\hline 002 & 17 & 16 & 16 & 18 & 19 & 14 & 16 & 18 & 17 & 1 & \\
\hline 003 & -29 & -24 & -26 & -32 & -36 & -24 & -27 & -27 & -17 & 5 & \\
\hline 004 & -3 & -2 & -2 & -3 & -4 & -5 & -3 & -2 & -2 & 1 & \\
\hline 005 & -6 & -4 & -5 & -7 & -9 & -20 & -8 & -3 & 0 & 6 & \\
\hline 006 & 7 & 5 & 6 & 8 & 9 & 10 & 8 & 6 & 5 & 2 & \\
\hline 007 & 5 & 3 & 4 & 6 & 7 & 11 & 9 & 0 & -5 & 5 & \\
\hline 008 & -7 & -6 & -7 & -8 & -9 & -6 & -6 & -8 & -8 & 1 & \\
\hline 009 & -17 & -16 & -17 & -18 & -20 & -4 & -19 & -15 & -12 & 5 & \\
\hline 010 & 3 & 2 & 2 & 3 & 4 & 3 & 3 & 3 & 3 & 1 & \\
\hline Mean & -5 & -4 & -5 & -5 & -6 & -4 & -5 & -4 & -3 & 1 & \\
\hline $\mathrm{SD}$ & 14 & 12 & 13 & 15 & 16 & 14 & 14 & 12 & 10 & & 13 \\
\hline \multicolumn{12}{|l|}{ Average } \\
\hline SD & & & & & & & & & & 3 & \\
\hline \multicolumn{12}{|c|}{ Single-leg Stance } \\
\hline & & & & & & & & & & \multicolumn{2}{|c|}{ Average } \\
\hline Participant & Standard & $-10 \mathrm{ML}$ & $-5 \mathrm{ML}$ & $+5 \mathrm{ML}+$ & $10 \mathrm{ML}$ & $-10 \mathrm{AP}$ & $-5 \mathrm{AP}$ & $+5 \mathrm{AP}$ & $+10 \mathrm{AP}$ & $\mathrm{SD}$ & SD \\
\hline 001 & -0.1 & 4 & 1 & -1 & -10 & 10 & 13 & -3 & -4 & 7 & \\
\hline 002 & 7 & -4 & -1 & 11 & 15 & 3 & 7 & -5 & -13 & 9 & \\
\hline 003 & -54 & -21 & -36 & -62 & -56 & -28 & -41 & 11 & 55 & 37 & \\
\hline 004 & 12 & 7 & 10 & 16 & 31 & 8 & 13 & 6 & -17 & 13 & \\
\hline 005 & -1 & 6 & 4 & 6 & 14 & 3 & 3 & 2 & 3 & 4 & \\
\hline 006 & -19 & -17 & -20 & -14 & -13 & -10 & -19 & 6 & 5 & 10 & \\
\hline 007 & 26 & 13 & 19 & 37 & 48 & 15 & 26 & -27 & -24 & 25 & \\
\hline 008 & -7 & -8 & -8 & -7 & 10 & -6 & -8 & -13 & -13 & 7 & \\
\hline 009 & -2 & -1 & -2 & -5 & -13 & 4 & 0 & -3 & -1 & 5 & \\
\hline 010 & -18 & -10 & -13 & -26 & -25 & -11 & -17 & -8 & -4 & 8 & \\
\hline Mean & -5 & -3 & -5 & -5 & 0.2 & -1 & -2 & -4 & -1 & 2 & \\
\hline $\mathrm{SD}$ & 22 & 11 & 16 & 27 & 30 & 13 & 20 & 11 & 22 & & 19 \\
\hline \multicolumn{12}{|l|}{ Average } \\
\hline SD & & & & & & & & & & 11 & \\
\hline
\end{tabular}


Table 3: Coefficient of determinations $\left(\mathrm{R}^{2}\right)$ for the linear regression of the strength estimates and total hip (TH), femoral neck (FN), and trochanter (Troch) DXA-derived vBMD measurements. *indicates statistical significance $(\mathrm{p}<0.05)$.

\begin{tabular}{|c|c|c|c|c|c|c|c|c|}
\hline \multicolumn{9}{|c|}{ Linear Regression } \\
\hline & \multicolumn{4}{|c|}{ Single-Leg Stance } & \multicolumn{4}{|c|}{ Sideways Fall } \\
\hline DXA Parameter & $\mathrm{R}^{2}$ & Adj. $\mathrm{R}^{2}$ & RMSE & p-value & $\mathrm{R}^{2}$ & Adj. $R^{2}$ & RMSE & p-value \\
\hline TH integral vBMD & 0.19 & 0.15 & 526 & $0.03 *$ & 0.09 & 0.05 & 125 & 0.14 \\
\hline FN integral vBMD & 0.12 & 0.08 & 548 & 0.09 & 0.09 & 0.05 & 125 & 0.14 \\
\hline Troch integral vBMD & 0.06 & 0.02 & 566 & 0.23 & 0.06 & 0.02 & 127 & 0.22 \\
\hline TH trabecular vBMD & 0.22 & 0.18 & 516 & $0.02 *$ & 0.03 & -0.009 & 129 & 0.39 \\
\hline FN trabecular vBMD & 0.12 & 0.08 & 547 & 0.08 & 0.02 & -0.02 & 130 & 0.49 \\
\hline Troch trabecular vBMD & 0.09 & 0.05 & 556 & 0.14 & 0.04 & -0.001 & 128 & 0.33 \\
\hline TH cortical vBMD & 0.003 & -0.04 & 582 & 0.80 & 0.02 & -0.02 & 130 & 0.47 \\
\hline FN cortical vBMD & 0.001 & -0.04 & 583 & 0.86 & 0.04 & -0.003 & 129 & 0.35 \\
\hline Troch cortical vBMD & 0.005 & -0.04 & 582 & 0.73 & 0.03 & -0.008 & 129 & 0.38 \\
\hline
\end{tabular}


Appendix 2: Linear regression of the strength estimates for single-leg stance (SLS) and sideways fall (SF) with all DXA-derived vBMD measurements from the software for the HiRIT exercise group.

\begin{tabular}{|c|c|c|c|c|c|c|}
\hline \multirow[t]{2}{*}{ HiRIT } & \multicolumn{2}{|l|}{ SLS } & \multicolumn{2}{|l|}{$\mathrm{SF}$} & \multirow[t]{2}{*}{ SLS } & \multirow[t]{2}{*}{$\mathrm{SF}$} \\
\hline & $\mathrm{R} 2$ & $\mathrm{p}$ & $\mathrm{R} 2$ & $\mathrm{p}$ & & \\
\hline '"sdens Total (mg/cm2)"' & -0.016 & 0.443 & 0.010 & 0.276 & FALSE & FALSE \\
\hline "'vbmd Trabecular Total (mg/cm3) & 0.183 & 0.017 & -0.009 & 0.387 & TRUE & FALSE \\
\hline '"vbmd Integral Total (mg/cm3)"' & 0.151 & 0.028 & 0.050 & 0.140 & TRUE & FALSE \\
\hline "'bmc Integral Total (g)"' & 0.096 & 0.068 & -0.027 & 0.565 & FALSE & FALSE \\
\hline "'bmc Integral Neck (g)"' & 0.310 & .002 & -0.036 & 0.717 & TRUE & FALSE \\
\hline '"bmc Integral Troch (g)"' & -0.042 & 0.954 & -0.020 & 0.482 & FALSE & FALSE \\
\hline "'bmc Integral Shaft (g)"' & 0.109 & 0.055 & -0.035 & 0.701 & FALSE & FALSE \\
\hline '"bmc Trabecular Total (g)"' & 0.137 & 0.035 & -0.039 & 0.809 & TRUE & FALSE \\
\hline '"bmc Trabecular Neck (g)"' & 0.295 & 0.002 & -0.042 & 0.996 & TRUE & FALSE \\
\hline "'bmc Trabecul & -0.036 & 0.729 & -0.033 & 0.665 & FALSE & FALSE \\
\hline '"bmc Trabecular Shaft & 0.150 & 0.029 & -0.041 & 0.895 & TRUE & FALSE \\
\hline "'bmc Cortical Total (g)"' & 0.006 & 0.297 & -0.022 & .505 & FALSE & FALSE \\
\hline "'bmc Cortical Neck (g)"' & 0.096 & 0.068 & -0.019 & 0.476 & FALSE & FALSE \\
\hline '"bmc Cortical Troch (g)"'" & -0.040 & 0.826 & -0.019 & 0.473 & FALSE & FALSE \\
\hline '"bmc Cortical Shaft (g)"' & 25 & 0.211 & -0.033 & 666 & ALSE & FALSE \\
\hline '"vbmd Integral Neck (1 & 0.079 & 0.088 & 0.049 & 0.144 & ALSE & FALSE \\
\hline '"vbmd Integral Troch (mg & 0.019 & 0.234 & 0.023 & .221 & FALSE & FALSE \\
\hline '"vbmd Integral Shaft (mg/ & .123 & .044 & 0.022 & 0.223 & TRUE & FALSE \\
\hline '"vbmd Trabecular Neck (mg/cm3)"' & 0.084 & 0.083 & -0.020 & 0.486 & FALSE & FALSE \\
\hline "'vbmd Trabecular Troch ( & 0.052 & 0.136 & -0.001 & 0.330 & FALSE & FALSE \\
\hline '"vbmd Trabe & 99 & 0.025 & -0.023 & 0.514 & TRUE & FALSE \\
\hline '"vbmd Cortical Total (1 & -0.039 & 0.804 & -0.019 & 0.469 & FALSE & FALSE \\
\hline '"vbmd Cortical Neck (r & -0.040 & 358 & -0.003 & .349 & ALSE & FALSE \\
\hline "'vbmd Cortical Troch ( & -0.036 & 0.729 & -0.008 & 0.380 & ALSE & FALSE \\
\hline '"vbmd Cortical S & -0.041 & 0.933 & -0.027 & 0.569 & FALSE & FALSE \\
\hline '"volume Integral Total (c & -0.013 & 0.420 & -0.023 & 0.519 & FALSE & FALSE \\
\hline '"volume Integral Neck (cm3)"' & 0.134 & 0.037 & -0.026 & 0.555 & TRUE & FALSE \\
\hline '"volume Integral Troch & 1 & 226 & -0.030 & 604 & ALSE & FALSE \\
\hline '"volume Integral Shaft (c & 4 & 0.182 & -0.032 & 0.633 & FALSE & FALSE \\
\hline '"volume Trabecular Total & -0.030 & 0.608 & -0.006 & 0.368 & FALSE & FALSE \\
\hline '"volume Trabecular Neck (cm3)"' & 0.115 & 0.050 & -0.016 & 0.443 & ALSE & FALSE \\
\hline '"volume Trabecular Troch (cm3)'" & 0.027 & 0.207 & -0.017 & 0.452 & FALSE & FALSE \\
\hline '"volume Trabecular Shaft (cm3)"' & 0.001 & 0.323 & -0.021 & 0.494 & FALSE & FALSE \\
\hline '"volume Corti & 1 & 0.192 & -0.036 & 0.732 & FALSE & FALSE \\
\hline '"volume Cortical Neck (cm3)"' & 35 & 0.036 & -0.039 & 0.802 & TRUE & FALSE \\
\hline '"volume Cortical Troch (cm3)"' & -0.038 & 0.759 & -0.032 & 0.637 & FALSE & FALSE \\
\hline '"volume Cortical Shaft (cm3)"' & 0.091 & 0.074 & -0.041 & 0.885 & FALSE & FALSE \\
\hline "'mct Total (mm)"' & -0.003 & 0.346 & -0.004 & 0.351 & FALSE & FALSE \\
\hline "'mct Nec & & & 0.015 & 0.251 & FALSE & FALSE \\
\hline "'mct Neck Lat (mm)"' & -0.031 & 0.624 & 0.133 & 0.038 & FALSE & TRUE \\
\hline (mm)"' & & 0.295 & -0.042 & 0.957 & FALSE & FALSE \\
\hline '"mct InterTroch (mm)"' & -0.019 & 0.472 & -0.013 & 0.419 & FALSE & FALSE \\
\hline
\end{tabular}




\begin{tabular}{|c|c|c|c|c|c|c|}
\hline "mct InterTro & -0.041 & 0.912 & -0.019 & 0.474 & SE & - \\
\hline "'mct It & 0.028 & 0.201 & -0.042 & .967 & ALSE & ALSE \\
\hline '"mct Shaft_cort (mm)"' & 0.009 & 0.280 & -0.028 & 0.577 & FALSE & ALSE \\
\hline '"mct Shaft_cort Lat (mm)"' & -0.033 & 0.653 & -0.020 & .487 & ALSE & FALSE \\
\hline "'mct Shaft_cort Med (mm)"' & -0.021 & 0.488 & -0.022 & .502 & ALSE & ILSE \\
\hline "'sdens Neck & 0.016 & 0.249 & 0.041 & 0.164 & FALSE & FALSE \\
\hline '"sdens Neck Lat (r & -0.035 & 0.689 & 0.117 & 0.049 & FALSE & TRUE \\
\hline "'sdens N & -0.019 & 0.469 & -0.033 & 0.667 & FALSE & FALSE \\
\hline '"sdens InterTroch (mg/cm2)"' & -0.027 & 0.562 & 0.008 & 0.286 & FALSE & FALSE \\
\hline '"sdens InterTroch Lat (mg/cm2)"' & -0.041 & 0.876 & -0.009 & 0.388 & FALSE & FALSE \\
\hline "'sdens InterTroch Med (mg/cm & -0.016 & 0.444 & -0.029 & 0.595 & FALSE & FALSE \\
\hline "'sdens Shaft_cort (mg/cm2)"' & -0.015 & 0.433 & -0.017 & 0.450 & FALSE & FALSE \\
\hline "'sdens S & -0.032 & 0.646 & -0.034 & 0.680 & FALSE & FALSE \\
\hline '"sdens Shaft_ & -0.028 & 0.571 & -0.016 & 0.443 & FALSE & FALSE \\
\hline '"Neck SA (cm2)"' & 0.068 & 0.106 & -0.012 & 0.412 & FALSE & FALSE \\
\hline "'Neck CSA (cm2)"' & 0.226 & 0.008 & -0.039 & 0.815 & TRUE & FALSE \\
\hline '"Neck CSMI (cm4)"' & 0.226 & 0.008 & -0.036 & 0.722 & TRUE & FALSE \\
\hline '"Neck Z (cm3)"'" & 0.199 & 0.013 & -0.041 & 0.940 & TRUE & FALSE \\
\hline "'InterTroch SA (cm2)"' & -0.038 & 0.759 & -0.003 & 0.345 & FALSE & FALSE \\
\hline "'InterTroch CSA (cm2)"' & 0.100 & 0.064 & -0.027 & 0.563 & FALSE & FALSE \\
\hline "'InterTroch CSMI (cm4)"' & -0.006 & 0.365 & -0.038 & 0.772 & FALSE & FALSE \\
\hline "InterTroch Z (cm3)"' & -0.028 & 0.583 & -0.033 & 0.653 & FALSE & FALSE \\
\hline
\end{tabular}


Appendix 3: Proximal femoral strength estimations at baseline and 8-month follow-up in single-leg stance and sideways fall for the HiRIT exercise group.

\begin{tabular}{|c|c|c|c|c|c|c|c|c|}
\hline & \multicolumn{4}{|c|}{ SINGLE-LEG STANCE } & \multicolumn{4}{|c|}{ SIDEWAYS FALL } \\
\hline Participant & $\begin{array}{r}\text { BASELINE } \\
(\mathrm{N})\end{array}$ & $\begin{array}{r}\text { FOLLOW- } \\
\text { UP (N) }\end{array}$ & $\begin{array}{r}\text { DIFF } \\
(\mathrm{N})\end{array}$ & $\begin{array}{r}\text { DIFF } \\
(\%)\end{array}$ & $\begin{array}{r}\text { BASELINE } \\
(\mathrm{N})\end{array}$ & $\begin{array}{r}\text { FOLLOW- } \\
\text { UP (N) }\end{array}$ & $\begin{array}{r}\text { DIFF } \\
(\mathrm{N})\end{array}$ & $\begin{array}{r}\text { DIFF } \\
(\%)\end{array}$ \\
\hline 001 & 2384 & 3879 & 1495 & $63 \%$ & 956 & 954 & -2 & $-0.2 \%$ \\
\hline 002 & 2356 & 3024 & 667 & $28 \%$ & 864 & 904 & 40 & $5 \%$ \\
\hline 003 & 2603 & 2822 & 219 & $8 \%$ & 1280 & 1181 & -99 & $-8 \%$ \\
\hline 004 & 4423 & 4249 & -173 & $-4 \%$ & 1127 & 1149 & 22 & $2 \%$ \\
\hline 005 & 3433 & 3481 & 48 & $1 \%$ & 936 & 1064 & 128 & $14 \%$ \\
\hline 006 & 4004 & 4546 & 541 & $14 \%$ & 1230 & 1274 & 44 & $4 \%$ \\
\hline 007 & 4341 & 4575 & 234 & $5 \%$ & 1133 & 1108 & -25 & $-2 \%$ \\
\hline 008 & 3413 & 2988 & -425 & $-12 \%$ & 615 & 1023 & 408 & $66 \%$ \\
\hline 009 & 3219 & 4037 & 818 & $25 \%$ & 1148 & 992 & -156 & $-14 \%$ \\
\hline 010 & 4262 & 3831 & -431 & $-10 \%$ & 1690 & 1584 & -106 & $-6 \%$ \\
\hline 011 & 4163 & 3751 & -412 & $-10 \%$ & 1048 & 1094 & 46 & $4 \%$ \\
\hline 012 & 3626 & 4443 & 817 & $23 \%$ & 913 & 833 & -80 & $-9 \%$ \\
\hline 013 & 3915 & 4620 & 705 & $18 \%$ & 1384 & 1284 & -100 & $-7 \%$ \\
\hline 014 & 4071 & 3044 & -1027 & $-25 \%$ & 1180 & 984 & -195 & $-17 \%$ \\
\hline 015 & 4200 & 4187 & -12 & $0 \%$ & 1444 & 1389 & -55 & $-4 \%$ \\
\hline 016 & 4123 & 3900 & -222 & $-5 \%$ & 1169 & 1213 & 44 & $4 \%$ \\
\hline 017 & 3851 & 3982 & 131 & $3 \%$ & 851 & 636 & -215 & $-25 \%$ \\
\hline 018 & 3866 & 3391 & -475 & $-12 \%$ & 1443 & 1331 & -113 & $-8 \%$ \\
\hline 019 & 3749 & 4535 & 785 & $21 \%$ & 951 & 779 & -172 & $-18 \%$ \\
\hline 020 & 3792 & 3645 & -147 & $-4 \%$ & 1070 & 1360 & 290 & $27 \%$ \\
\hline 021 & 2388 & 2078 & -310 & $-13 \%$ & 865 & 852 & -13 & $-1 \%$ \\
\hline 022 & 3127 & 2828 & -299 & $-10 \%$ & 879 & 1030 & 151 & $17 \%$ \\
\hline 023 & 3355 & 3347 & -8 & $-0.2 \%$ & 975 & 1042 & 67 & $7 \%$ \\
\hline 024 & 2360 & 3491 & 1131 & $48 \%$ & 917 & 1119 & 202 & $22 \%$ \\
\hline 025 & 4644 & 4549 & -94 & $-2 \%$ & 887 & 774 & -112 & $-13 \%$ \\
\hline 026 & 3003 & 2936 & -67 & $-2 \%$ & 1227 & 1044 & -182 & $-15 \%$ \\
\hline 027 & 2471 & 2527 & 57 & $2 \%$ & 1049 & 1134 & 86 & $8 \%$ \\
\hline
\end{tabular}




\section{CONFLICT OF INTEREST STATEMENT}

Prof. Beck is the Director of The Bone Clinic. The other authors have nothing to disclose. 